\title{
Indice fisicoquímico de la calidad de agua para el manejo de lagunas tropicales de inundación
}

\author{
Ana Gabriela Pérez-Castillo ${ }^{1,2}$ \& Alexis Rodríguez ${ }^{2}$ \\ 1. Escuela de Química, Universidad de Costa Rica,2060 San José, Costa Rica; holagaby@gmail.com \\ 2. Centro de Investigación en Contaminación Ambiental, Universidad de Costa Rica, 2060 San José, Costa Rica; \\ alrodri@racsa.co.cr
}

Recibido 30-VIII-2007. Corregido 16-VI-2008. Aceptado 16-VII-2008.

\begin{abstract}
Physicochemical water quality index, a management tool for tropical-flooding lagoons. We propose ICA-L, a wetland physicochemical water quality index (WWQI), to be used as a management tool for seasonal-flooding lagoons in Palo Verde National Park, Guanacaste, Costa Rica. The goal is to preserve their natural role for native plants as well as migrants and local animal species. The index was developed in four steps: parameter selection, assignment of parameter weight, transformation of data to their corresponding sub indices and selection of an appropriate aggregation function. In this process, the following criteria were used as a reference: WQI from the National Sanitation Foundation, WQI for the Des Moines River, Escribano and De Frutos WQI, the international legislation on maximum acceptable concentration for different water quality variables, and the authors' personal criteria. The index includes the following parameters: dissolved oxygen percent saturation, $\mathrm{pH}$, nitrate concentration, total phosphorus concentration, chemical oxygen demand, concentration of suspended solids, electrical conductivity and temperature. The index sets itself to zero if the concentration of some toxic substance exceeds the maximum allowed limit. The adjustment values were based on "weights" defined in the National Sanitation Foundation Water quality Index (ICA-NSF). In this study, the weight of fecal coliforms count was excluded, the values of turbidity and the one for total solids were integrated into one (suspended solids) and a factor of 0.08 was assigned to the conductivity parameter. The sub indices associated to suspended solids were obtained from the quality of Kahler-Royer variation graph; the values for $\mathrm{pH}$ and the nitrate concentration from the graphs constructed for ICA-NSF. The percentage of dissolved oxygen saturation, in sites like irrigation channels, was evaluated directly from the quality variation graph constructed for ICANSF, whereas the same parameter for the flooding lagoons required an adjustment based on the optimal value for similar non contaminated ecosystems. The conductivity was evaluated from adjustments in the qualification functions commented by Escribano \& De Frutos. Chemical oxygen demand, total phosphorus and temperature, were qualified based on the functions developed for the ICA-L. Rev. Biol. Trop. 56 (4): 1905-1918. Epub 2008 December 12 .
\end{abstract}

Key words: water quality index, freshwater tropical system, wetland, seasonal flooding lagoons, physicochemical index, Palo Verde.

Los sistemas tropicales con agua dulce estacional (pantanos, esteros y charcas estacionales, llanuras inundables, lagunas de carga y descarga de acuíferos y humedales boscosos de agua dulce), como los del Parque Nacional Palo Verde (PNPV), en Guanacaste, Costa Rica, son ecosistemas muy productivos. Su biomasa es importante para la conservación de numerosas especies vegetales y animales. Contribuyen al desarrollo sostenible de la zona al: preservar una alta biodiversidad en flora y fauna silvestres (inclusive de especies migratorias), ofrecer protección contra la erosión, mitigar las inundaciones $\mathrm{y}$, favorecer la recarga de acuíferos subterráneos, la purificación del agua y la estabilización de las condiciones de lluvia y temperatura locales. Además, generan beneficios económicos como un hábitat rico para el 
crecimiento de peces, la posibilidad de pastoreo estacional de ganado y el impulso de opciones de recreación y turismo (UICN 2002).

En 1991, el sistema de lagunas estacionales de Palo Verde, fue designado sitio de importancia internacional por la Convención de Humedales Ramsar (Ramsar 1998) y en el año 2000, se estableció en su colindancia el sector de regadío de Tamarindo (SRT), como parte del Distrito de Riego Arenal - Tempisque (DRAT). El SRT ha contribuido al desarrollo agrícola, pero puede llegar a ser una amenaza, porque sus aguas de escorrentía y drenaje ingresan a la laguna La Bocana dentro del PNPV.

El riesgo de contaminación asociado con las actividades antropogénicas en el SRT, genera la necesidad de administrar los caudales y de idear un instrumento, para dar seguimiento a la calidad de las aguas en los canales de retorno y en la citada laguna y así, prevenir o aminorar modificaciones graves en este ecosistema. La importancia de este instrumento es significativa, porque adicionalmente, sería util para evaluar y comparar otras lagunas de inundación en el trópico.

La aplicación de índices de calidad del agua (ICA), es una metodología que aporta información reproducible sobre los atributos del agua $y$, una alternativa para dictaminar un cuerpo de agua sin recurrir a recopilaciones estadísticas de las tendencias, variable por variable y sitio por sitio.

Los ICA resumen y simplifican, en un único valor numérico, el cúmulo de información disponible sobre la calidad del agua. Estos índices facilitan el manejo de datos, evitan que las fluctuaciones en las mediciones invisibilicen las tendencias ambientales y permiten comunicar, en forma simple y veraz, la condición del agua para un uso deseado o efectuar comparaciones temporales y espaciales entre cuerpos de agua (House 1990, Alberti y Parker 1991). Por lo tanto, resultan útiles o accesibles para las autoridades políticas y el público en general.

Los ICA presentan desventajas como la pérdida de información respecto a las variables individuales y su interacción, la falta de ajuste a diferentes tipos de ecosistemas y la sensibilidad de los resultados a la forma en que el índice fue elaborado (problemas de ambigüedad, eclipsamiento y rigidez) (Swamee y Tyagi 2007).

Existen ICA construidos a partir de parámetros fisicoquímicos, que varían según la naturaleza del cuerpo de agua, el posible uso del agua, las condiciones climáticas y geológicas de la región y el criterio de expertos (Couillard y Lefebvre 1985, House 1990, Boyacioglu 2007). Los datos físicoquímicos permiten analizar las causas del problema, pero exigen una frecuencia de muestreo apropiada, pues indican una condición puntual del agua. Además, está el grupo de índices biológicos de calidad del agua, en los cuales se registra la abundancia y la diversidad de ciertos organismos, especialmente macroinvertebrados bentónicos. Éstos permiten, a partir de su tolerancia particular a la contaminación, estimar el efecto acumulado de las intervenciones humanas en el ambiente a lo largo del tiempo (Astorga 1994, Figueroa et al. 2007).

En general, el desarrollo de ICA se ha enfocado principalmente al estudio de ríos o corrientes y falta investigación sobre lagunas de inundación, donde la diversidad en la naturaleza de estos ecosistemas dificulta el desarrollo de índices biológicos. Particularmente, en las lagunas con un bajo porcentaje de oxígeno disuelto y limitada biodiversidad de organismos, los índices fisicoquímicos pueden ser un instrumento más confiable para determinar el ingreso de agentes contaminantes.

En la zona del DRAT, se han evaluado las corrientes de agua dulce y de los canales mediante el índice de calidad del agua para la vida acuática (ICA-VA) (RodríguezUlloa 1996) y varios índices biológicos (RizoPatrón 2003). El ICA-VA, obtenido a partir de variables fisicoquímicas, no considera las particularidades asociadas con el estudio de ecosistemas como las lagunas de inundación de agua dulce, pues se diseñó para el seguimiento de los ríos afectados por el distrito de riego. De los índices biológicos, resultó más apropiado el Biological Monitoring Working Party modificado por Springer, para las familias de 
macroinvertebrados presentes en Costa Rica. Sin embargo, éste índice diseñado para la evaluación de ríos, aunque efectivo en la evaluación de los canales de retorno del regadío, no tuvo un desempeño adecuado para predecir los efectos de las actividades productivas, en las lagunas de inundación con un porcentaje de saturación de oxígeno bajo, donde la biodiversidad de macroinvertebrados bentónicos era reducida (Rizo-Patrón 2003).

El objetivo de este trabajo fue diseñar y construir un ICA fisicoquímico, como instrumento para evaluar la condición del agua de las lagunas de inundación de agua dulce tropicales y de los canales de regadío, para el sostenimiento de la biodiversidad y el desarrollo de la vida acuática, cuando se tienen riesgos de degradación debidos a prácticas agropecuarias.

\section{MATERIALES Y MÉTODOS}

Para construir el índice de calidad del agua para el manejo de lagunas de inundación de agua dulce (ICA-L), se siguió el siguiente procedimiento: selección de las variables por analizar, definición de su ponderación, transformación de las diferentes mediciones en un puntaje relacionado con la calidad del agua y la integración de los puntajes en un valor numérico o índice, que se asocia con una categoría o juicio acerca de la utilidad del agua. Los puntajes de calidad se obtuvieron a partir del uso de gráficas de variación de la calidad o la creación de funciones de calificación.

En la selección y ponderación de las variables del ICA-L y en la estimación de cada puntaje, se consideraron las siguientes referencias: el índice de calidad del agua de la National Sanitation Foundation (ICA-NSF) modificado por Deininger (1980); el ICA desarrollado para la cuenca del río Des Moines River en el centro de Iowa (Kahler-Royer 1999); el ICA para aguas superficiales comentado por Escribano y De Frutos (1987) y su relación con los efectos antropogénicos; la comparación con los límites máximos permitidos en el agua, en función de su uso para sostener la vida acuática definidos en legislación internacional (EPA 1986, 2000,
2002) y los criterios desarrollados en el transcurso de la investigación.

Selección de las variables y manejo de sustancias tóxicas: Las variables se escogieron considerando como prioridad el sostenimiento de la biodiversidad y el desarrollo de la vida acuática en el cuerpo de agua, como factores de riesgo las prácticas agropecuarias (Rickert 1993) y como ecosistema meta, las lagunas tropicales de inundación y los canales de regadío cercanos. A partir de estos criterios se seleccionaron las siguientes variables: porcentaje de saturación de oxígeno disuelto $\left(\mathrm{st}_{2}\right)$, concentración de sólidos suspendidos ( $\mathrm{SS}$ ), $\mathrm{pH}$, concentración de nitratos $\left(\mathrm{NO}_{3}\right)$, concentración de fósforo total (P total), demanda química de oxígeno (DQO), conductividad eléctrica $(\mathrm{Ct})$ y temperatura (T). Además, el índice se fija automáticamente en cero, si la concentración de alguna sustancia tóxica excede el límite máximo permitido.

Factores de ponderación: La ponderación de cada variable incorporada corresponde a su repercusión en la capacidad del agua para sostener la vida acuática en las lagunas de inundación. Estos factores de ponderación se ajustaron con base en los pesos del ICA-NSF mencionados por Deininger (1980), excluyéndose el peso del conteo de coliformes fecales (0.16). Además, las ponderaciones individuales de la turbiedad (0.07) y de los sólidos totales (0.08) se integraron en la de los sólidos suspendidos. En el caso de la conductividad se asignó un factor de 0.08. Finalmente, la diferencia con la unidad (0.08) se distribuyó de manera proporcional entre los factores de ponderación establecidos como punto de partida.

Funciones de calificación: Los puntajes asociados con los sólidos suspendidos se obtuvieron de la gráfica de variación de la calidad expuesta por Kahler-Royer (1999). Las valoraciones del $\mathrm{pH}$ y de la concentración de nitratos se extrajeron de las gráficas construidas para el ICA-NSF (Deininger 1980).

El porcentaje de saturación del oxígeno disuelto, en sitios como los canales del regadío, 
se evaluó directamente de la gráfica de variación de la calidad construida para el ICA-NSF; en tanto que, para las lagunas de inundación se requirió hacer ajustes a dicha gráfica, con base en el valor óptimo para ecosistemas similares no expuestos a contaminación.

La conductividad se evaluó a partir de ajustes en las funciones de calificación comentadas por Escribano y De Frutos (1987). La demanda química de oxígeno, el fósforo total y la temperatura, se calificaron con base en funciones desarrolladas para el ICA-L.

\section{RESULTADOS}

Gráficas de calidad y funciones de calificación desarrolladas para el ICA-L: La calidad del agua para el manejo de lagunas de inundación en relación con la demanda química de oxígeno y el fósforo total, se analizó con base en las concentraciones límite establecidas a partir de criterios de sostenibilidad ambiental y, la temperatura, de acuerdo con la adaptabilidad de las especies a las condiciones ambientales del ecosistema de interés.
En el Cuadro 1 se resumen los puntos y los criterios de evaluación escogidos para las tres variables citadas. Con los puntos definidos para cada variable se generó una curva de mejor ajuste (Fig. 1). Estas curvas se dibujaron siguiendo el perfil de las gráficas de calidad de la demanda biológica de oxígeno (DBO) y de la concentración de fosfatos que se desarrollaron al crear el ICA-NSF y, una distribución normal para la temperatura. Lo anterior, permitió deducir las funciones de calificación que se presentan en el Cuadro 2.

Las funciones de calificación se utilizan para calcular el puntaje asociado con cada medición efectuada en los puntos de muestreo, dentro del ámbito de aplicación indicado para cada variable.

Ecuación de cálculo del ICA-L: La integración del valor numérico del ICA-L se efectúo a partir del cálculo de un producto ponderado y se evaluó con base en los puntajes de las variables incluidas, definidos dentro de una escala de uno a cien, según la ecuación:

$$
\left.I C A-L=\left(Q_{s t}\right)_{2}\right)^{0.18} \times\left(Q_{S S}\right)^{0.16} \times\left(Q_{p H}\right)^{0.12} \times\left(Q_{D Q O}\right)^{0.12} \times\left(Q_{N O_{3}}\right)^{0.11} \times\left(Q_{P \text { total }}\right)^{0.11} \times\left(Q_{T}\right)^{0.11} \times\left(Q_{C t}\right)^{0.09}
$$

Donde Q, corresponde al puntaje de cada propiedad designada por el subíndice.

\section{CUADRO 1}

Criterios de evaluación y puntajes definidos para la deducción de las funciones de calificación $(Q)$ de las variables $D Q O$, fósforo total y temperatura

TABLE 1

Evaluation criteria and subindices defined for the deduction of qualification functions (Q) of DQO, total phosphorus and temperature variables

\begin{tabular}{|c|c|c|c|c|c|c|c|}
\hline \multirow{2}{*}{ Variable } & \multicolumn{7}{|c|}{ Categoría } \\
\hline & Óptima & Excelente & Buena & Regular & Mala & Pésima & No apta \\
\hline $\begin{array}{l}\mathrm{DQO}\left(\mathrm{mg} \mathrm{O}_{2} / 1\right) \\
\text { (Puntaje) }\end{array}$ & -- & $\begin{array}{l}\leq 25 \\
(100)\end{array}$ & $\begin{array}{l}\text { De } 26 \\
\text { (De } 85\end{array}$ & $\begin{array}{l}40 \\
45)\end{array}$ & $\begin{array}{l}\text { De } 41 \text { a } 60 \\
(\text { De } 44 \text { a } 25)\end{array}$ & $\begin{array}{l}\text { De } 61 \text { a } 150 \\
\text { (De } 24 \text { a } 1.1)\end{array}$ & $\begin{array}{c}>151 \\
(1, \text { si } \mathrm{DBO}>15)\end{array}$ \\
\hline $\begin{array}{l}\text { P total }(\mu \mathrm{g} / 1) \\
\text { (Puntaje) }\end{array}$ & -- & $\begin{array}{l}\leq 50 \\
(100)\end{array}$ & -- & $\begin{array}{l}\mathrm{De} \\
(\mathrm{De}\end{array}$ & $\begin{array}{l}1 \text { a } 100 \\
70 \text { a } 26)\end{array}$ & $\begin{array}{l}\text { De } 101 \text { a } 150 \\
(\text { De } 25 \text { a } 2)\end{array}$ & $\begin{array}{l}>151 \\
(1)\end{array}$ \\
\hline $\begin{array}{l}\text { Temperatura }\left({ }^{\circ} \mathrm{C}\right) \\
\text { (Puntaje) }\end{array}$ & $\begin{array}{c}27.2 \\
(100)\end{array}$ & $\begin{array}{l}28.6-25.8 \\
(95)\end{array}$ & $\begin{array}{c}30.0-24.4 \\
(85)\end{array}$ & -- & $\begin{array}{c}34.2-20.2 \\
(50)\end{array}$ & -- & $\begin{array}{l}>37 \\
(1)\end{array}$ \\
\hline
\end{tabular}



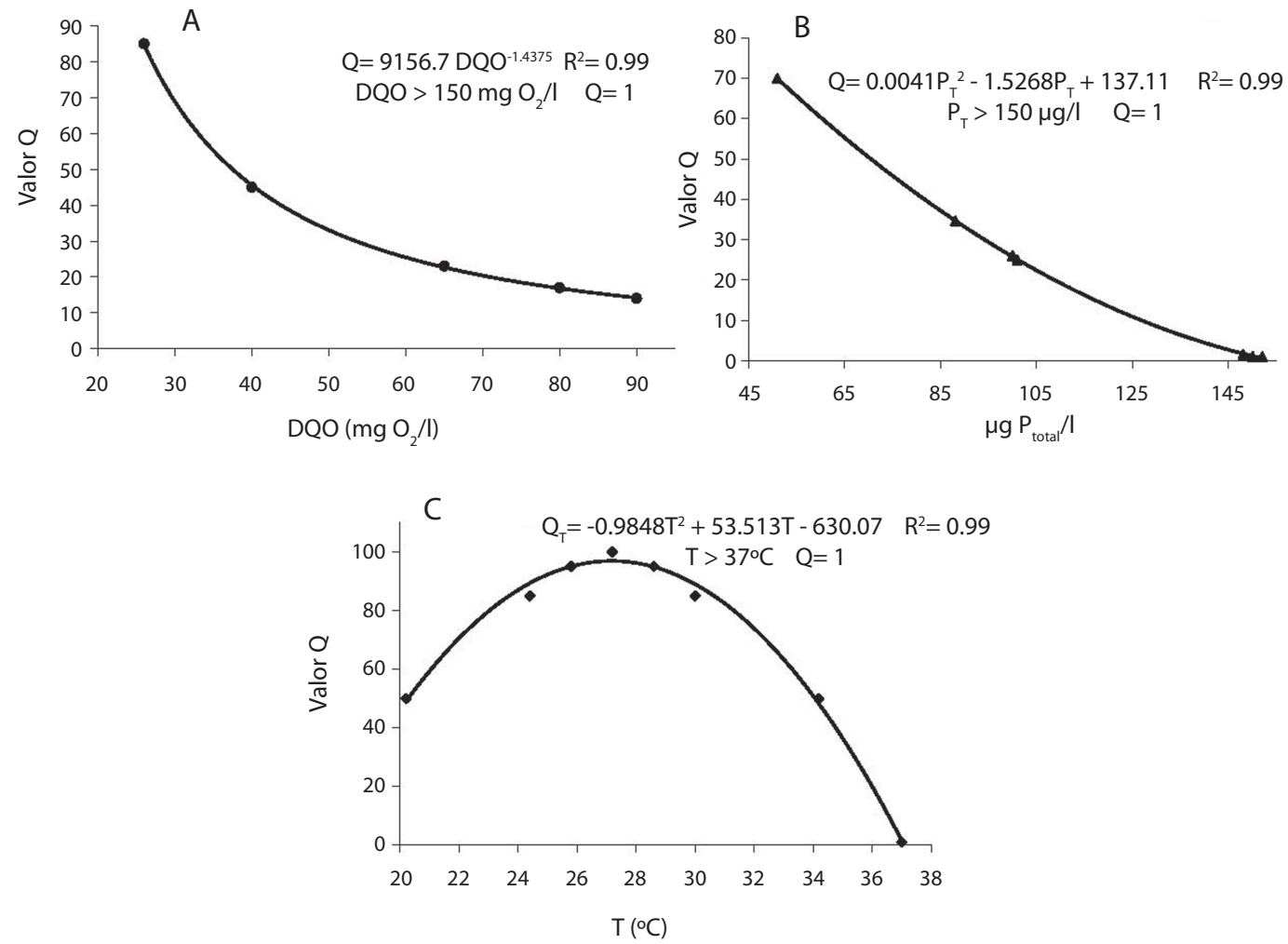

Fig. 1. Funciones de calificación obtenidas para el índice de calidad de agua para el manejo de lagunas de inundación. A: demanda química de oxígeno; B: concentración de fósforo total; C: temperatura.

Fig 1. Qualification functions obtained for the water quality index to manage freshwater flooding lagoons. A: chemical oxygen demand; B: total phosphorus concentration; C: temperature.

\section{CUADRO 2}

Funciones de calificación elaboradas de acuerdo con los parámetros del Cuadro 1

TABLE 2

Qualification functions elaborated according to the parameters from Table 1

Propiedad

Demanda química de oxígeno

Fósforo total

Temperatura
Función de calificación

$$
\begin{gathered}
\mathrm{Q}_{\mathrm{DQO}}=9156.7 \mathrm{DQO}^{(-1.4375)} \\
\mathrm{Q}_{\text {Ptotal }}=0.0041 \mathrm{P}_{\mathrm{T}}^{2}-1.5268 \mathrm{P}_{\mathrm{T}}+137.11 \\
\mathrm{Q}_{\mathrm{T}}=-0.9848 \mathrm{~T}^{2}+53.513 \mathrm{~T}-630.07
\end{gathered}
$$

Ámbito de aplicación

De 26 a $150 \mathrm{mg} \mathrm{O}_{2} / 1 \mathrm{DBO}>2 \mathrm{mg} \mathrm{O}_{2} / 1$

De 51 a $150 \mu \mathrm{g} / 1$

De 20 a $37^{\circ} \mathrm{C}$ 
La relación entre el ICA-L y la calidad del agua de las lagunas tropicales de inundación para el sostenimiento de la vida acuática, se resume en el Cuadro 3, que es una modificación de la propuesta de House (1990). Un ICA-L igual a cien define el agua como de excelente calidad y un resultado de uno, indica que el agua de la laguna de inundación no es apta para el mantenimiento de la vida acuática.

\section{DISCUSIÓN}

\section{Variables evaluadas según las gráficas de calidad de índices previos}

Porcentaje de saturación de oxígeno disuelto: Esta variable se incluye en éste y en otros índices con una alta ponderación, porque define en gran parte la biodiversidad y la supervivencia de la comunidad biótica.

La mayoría de los peces toleran una concentración baja de oxígeno disuelto por cierto período de tiempo. Sin embargo, reducciones por debajo del porcentaje de saturación generan efectos negativos sobre la biodiversidad, el crecimiento, la reproducción y la actividad de éstos.

El oxígeno disuelto determina si en los procesos de degradación dominan los organismos aerobios o los anaerobios, lo que marca la capacidad del agua para llevar a cabo procesos de autopurificación. Además, si su concentración es muy baja contribuye a que los organismos sean más susceptibles al envenenamiento con metales pesados y plaguicidas (Gaunt y Barker 2000).

La medición del oxígeno disuelto como porcentaje de saturación, facilita la comparación de sitios con distintas temperaturas, presiones o salinidades (Chapman 1996). La gráfica de calidad del porcentaje de saturación de oxígeno disuelto en el ICA-NSF se ajusta, en general, a la realidad de las especies tropicales, donde el porcentaje ideal para muchos peces es mayor a $67 \%$, mientras que las concentraciones de oxígeno disuelto menores a $55 \%$, afectan adversamente la biodiversidad y la supervivencia de la comunidad biótica. También, concentraciones superiores a $100 \%$ de saturación de oxígeno disuelto resultan peligrosas para la vida acuática, pues facilitan que burbujas de oxígeno bloqueen el flujo sanguíneo (Chapman 1996).

Sólidos suspendidos: Esta variable se incorporó en el ICA-L para evaluar el efecto sobre el agua, de la erosión ocasionada por

CUADRO 3

Puntaje del ICA-L y su relación con la calidad del agua para la vida acuática

TABLE 3

ICA-L values and its relation with water quality for aquatic life

\begin{tabular}{|c|c|c|}
\hline ICA-L & Categoría & Descripción de la calidad del agua \\
\hline $86-100$ & Excelente & $\begin{array}{l}\text { No presenta peligros para el ecosistema. Es adecuada para el desarrollo de todas las } \\
\text { especies. }\end{array}$ \\
\hline $71-85$ & Buena & $\begin{array}{l}\text { Sostiene una alta biodiversidad de vida acuática. Se presentan períodos donde algún } \\
\text { indicador muestra peligros para el ecosistema. En este caso, si la situación no mejora en } \\
\text { un período breve, se empezarían a ver cambios en la composición del ecosistema. }\end{array}$ \\
\hline $51-70$ & Regular & $\begin{array}{l}\text { Existen signos de contaminación, como aumento en la concentración de nutrimentos. Se } \\
\text { observa una reducción de la diversidad en los organismos acuáticos y un desequilibrio } \\
\text { en el crecimiento de algas y vegetación acuática. }\end{array}$ \\
\hline $26-50$ & Mala & $\begin{array}{l}\text { Sostiene una baja biodiversidad de vida acuática, principalmente de especies tolerantes. } \\
\text { Manifiesta problemas con fuentes de contaminación puntuales y no puntuales. }\end{array}$ \\
\hline $0-25$ & Pésima & $\begin{array}{l}\text { Posibilita el crecimiento de poblaciones elevadas de un limitado número de organismos } \\
\text { resistentes a aguas muy contaminadas. }\end{array}$ \\
\hline
\end{tabular}


las prácticas agrícolas y el acarreo de material durante la escorrentía de aguas de lluvia o de regadío. Los sólidos generan problemas de colmatación y, la sedimentación puede formar deltas aguas arriba del reservorio y hasta destruir hábitats para los organismos acuáticos al disminuir la columna de agua. Además, existe una estrecha relación entre la concentración de los sólidos suspendidos y la calidad del agua, debido a su capacidad de adsorción de contaminantes como plaguicidas y nutrimentos, al control que ejercen sobre la turbiedad del agua y a su absorción de calor que aumenta la temperatura del agua (Dagne et al. 2005).

El análisis de la concentración de los sólidos suspendidos es preferible a la de los sólidos sedimentables de otros índices, pues la disminución en la velocidad del flujo y la presencia de las plantas acuáticas en las lagunas de inundación, logran la remoción no sólo de los sólidos sedimentables, sino de hasta el $90 \%$ del material suspendido (Rodríguez 1995).

La integración de la ponderación individual de la turbiedad y la de los sólidos totales del ICA-NSF, para calcular la ponderación de los sólidos suspendidos, obedeció a su interrelación directa con la presencia de éstos y el efecto acumulativo ejercido sobre las lagunas de inundación.

pH: Al igual que el porcentaje de saturación del oxígeno disuelto, el $\mathrm{pH}$ es una variable común entre los ICA, por su potencial como indicador de la calidad del agua en general, del grado de afectación de ésta por agentes contaminantes y de la extensión de una estela de contaminación producida por la descarga de un efluente.

Los cambios en el $\mathrm{pH}$ pueden indicar el ingreso de fertilizantes, particularmente cuando se registran mediciones continuas junto con la conductividad del cuerpo de agua y; de procesos de eutrofización, si se asocian con los ciclos de fotosíntesis y respiración de las algas. Además, el $\mathrm{pH}$ afecta la toxicidad de algunos compuestos, como el amoníaco, al controlar su ionización, así como, la disponibilidad biológica de ciertos contaminantes, como los metales pesados.
La gráfica de calidad del pH del ICA-NSF muestra que sólo en el intervalo de 6.5 a 8.5, el agua es apropiada para la subsistencia de muchos sistemas biológicos. Valores mayores a 9.0 y menores de 5.8 producen limitaciones al desarrollo y a la físiología de los organismos acuáticos (Chapman 1996). Estas condiciones también se cumplen para la biota de las lagunas de inundación, lo que permitió su uso en el ICA-L.

Nitrato: La concentración de nitratos, se incluyó en el ICA-L para visibilizar el lavado de fertilizantes y por su capacidad para favorecer los procesos de eutrofización antropogénica. En algunas ocasiones, cuando se tienen $\mathrm{pH}$ básicos, puede resultar conveniente sustituir esta concentración por la suma de nitrato y nitrógeno amoniacal, particularmente en condiciones anaerobias (Cude 2001).

En el índice construido el nitrito se desprecia pues el ecosistema no se asocia a efluentes industriales o de aguas negras. Además, el nitrito se convierte a nitrato cuando el oxígeno disuelto alcanza concentraciones tan bajas como 0.3 mg/l (Fuentes y Massol-Deyá 2002).

La eutrofización antropogénica resulta de importancia en el ICA-L, por estar orientado al análisis de lagunas de inundación. El ion nitrato, en condiciones naturales, rara vez excede de $0.45 \mathrm{mg} \mathrm{NO} / 1$ (Chapman 1996), lo que concuerda con el estudio sobre lagos de Costa Rica de Horn y Haberyan (1993). Concentraciones superiores a $0.9 \mathrm{mg} \mathrm{NO} / 1$ en lagos, tienden a estimular el crecimiento de las algas e indicar una posible condición eutrófica y mayores a 20 $\mathrm{mg} \mathrm{NO} / 1$ alertan sobre efluentes contaminados (Chapman 1996). Por ello, se ajustó la curva de calidad del ICA-NSF (Deininger 1980) reduciendo de 1.7 a $1.0 \mathrm{mg} / \mathrm{l}$ la concentración de nitrato asociada con un valor de $\mathrm{QNO}_{3}=90$.

\section{Variables evaluadas según ajustes en funciones de calificación}

Porcentaje de saturación oxígeno disuelto en lagunas de inundación: El análisis del oxígeno disuelto con respecto a la calidad del agua es diferente si se trata de lagunas o de 
canales de regadío, porque en las ciénegas o lagos es normal encontrar una marcada disminución en el porcentaje de saturación del oxígeno conforme se desciende desde la capa superficial. Lo anterior, por la mayor demanda microbiana de oxígeno que surge de la acumulación de material biodegradable en el fondo y por lo lento del flujo del agua que limita la capacidad de intercambio gaseoso con el aire (Lampert y Sommer 1997).

En el caso específico del ICA-L, se contempló la condición normal del humedal en la función de calificación para el porcentaje de oxígeno disuelto. Con este criterio, la curva correspondiente del ICA-NSF se ajustó asignándole, en el eje $\mathrm{X}$ de la gráfica de variación de la calidad, a un porcentaje de cinco por ciento el puntaje óptimo de 100 (Fig. 2). Dicho valor se obtuvo del porcentaje de saturación de oxígeno disuelto promedio medido para el seno del agua de la laguna Palo Verde (5,0 $\pm 1,3 \%)$, ecosistema representativo que no está expuesto a la influencia de agentes contaminantes.

Conductividad: El ICA-L incorpora la conductividad porque sirve como señal de ingreso de fertilizantes inorgánicos y por su relación con las concentraciones relativas de los iones cloruro, sulfato y potasio. Al obtenerse in situ a muy bajo costo, es un factor que contribuye a dar sostenibilidad a un programa de seguimiento ambiental. Primavesi et al. (2002) señalan a la conductividad como una de las variables que mejor discriminan la calidad del agua, entre los puntos de muestreo y su grado de protección hacia alteraciones antropogénicas. En general, se ha empleado como señal para establecer una estela de contaminación alrededor de un punto de descarga. Se encuentra incluida por ejemplo, en el ICA de la comunidad de Madrid (Escribano y De Frutos 1987) y en el presentado por Said et al. (2004).

El puntaje de la conductividad del ICA-L se definió con base en las funciones de calificación comentadas por Escribano y De Frutos (1987): $\mathrm{Q}_{\text {Ст-1 }}=120-0.08$ Ст, de 250 a $1000 \mu \mathrm{S} /$ cm y $\mathrm{Q}_{\text {Ст-2 }}=80-0.04$ С , hasta $1500 \mu \mathrm{S} / \mathrm{cm}$. Para valores de conductividad mayores se estableció un puntaje mínimo de 20 .

Estas funciones se seleccionaron porque se ajustan adecuadamente a la línea base proyectada para el SRT, donde el valor de $250 \mu \mathrm{S} / \mathrm{cm}$ se asocia con agua no contaminada $\left(\mathrm{Q}_{\mathrm{Ct}}=100\right)$

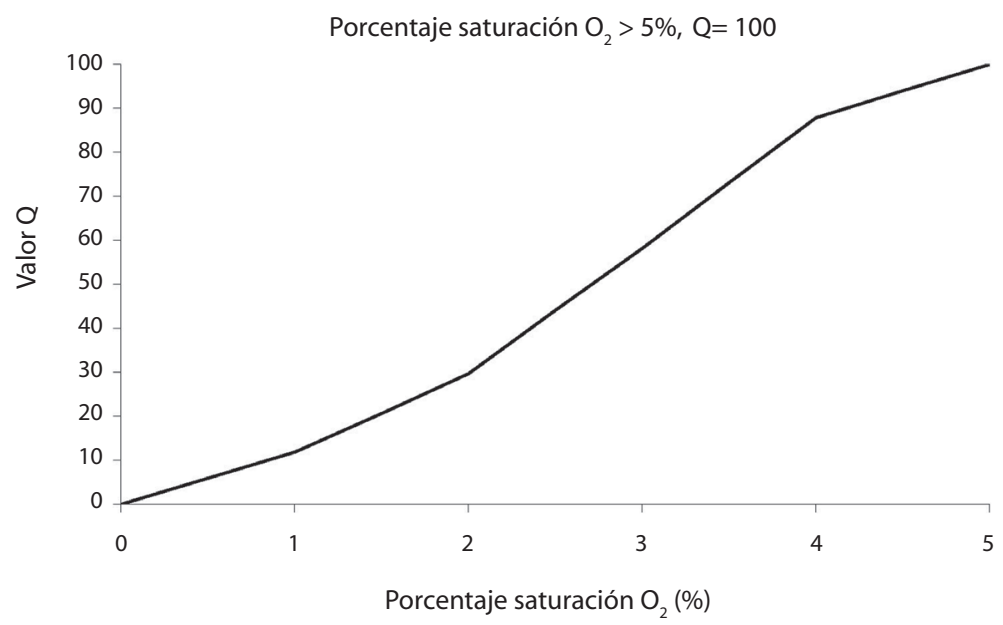

Fig. 2. Variación de la calidad del agua con el porcentaje de saturación del oxígeno presente en lagunas de inundación de agua dulce, con base en la curva análoga del índice de calidad de la National Sanitation Foundation.

Fig. 2. Variation of water quality with the dissolved oxygen saturation percentage present in freshwater flooding lagoons, adjusted from the analogous curve of the National Sanitation Foundation water quality index. 
y, el límite inferior para asignar al agua la categoría regular $\left(\mathrm{Q}_{\mathrm{Ct}}=60\right)$ corresponde a la conductividad de $750 \mu \mathrm{S} / \mathrm{cm}$, que coincide según Escribano y De Frutos (1987), con el límite máximo para el desarrollo apropiado de la piscicultura. Límite asociado al sostenimiento de la vida acuática, uso pensado para el ICA-L.

El valor asignado a conductividades superiores a $1500 \mu \mathrm{S} / \mathrm{cm}$ se fijó considerando el ICA-NSF, donde el valor de $\mathrm{Q}_{\mathrm{SD}}$ permanece constante a partir de una concentración de sólidos disueltos (SD) de $500 \mathrm{mg} / \mathrm{l}$ (Deininger 1980, Kahler-Royer 1999). Dicho valor de conductividad, no corresponde exactamente al valor que se obtendría con un factor de conversión respecto a los sólidos disueltos entre 0.55 y 0.75 (García et al. 2001), debido a que la posibilidad de un fenómeno de eutrofización antropogénica, se evalúa también en otros factores. A diferencia de otras variables, que ejercen un efecto más pronunciado en la calidad del agua al alejarse su concentración del parámetro recomendado, se fijó el puntaje mínimo en 20, pues las consecuencias sobre la calidad del agua no se acentúan a partir de cierta cantidad de sales disueltas.

\section{Variables evaluadas según criterios desarrollados para el ICA-L}

Temperatura: Se incluyó en el ICA-L debido a que afecta los procesos físicos (volatilización, solubilidad de sales y gases como el oxígeno, estratificación de estanques), los procesos químicos $(\mathrm{pH}$, equilibrio de ionización o concentración de amoníaco, velocidades de reacción) y los procesos biológicos (tasa metabólica, descomposición de materia orgánica) y por lo tanto, repercute en el efecto de los agentes contaminantes. $\mathrm{Al}$ asociarse el índice al estudio de humedales poco profundos en una zona tropical y afectada por áreas de regadío, se favorece la medición ocasional de valores extremos.

En el ICA-L se considera más importante el valor absoluto de la temperatura del cuerpo de agua que su cambio entre los sitios de medición, porque generalmente los muestreos se efectuaron en un intervalo de varias horas, o inclusive de un día para otro, con alteraciones en la nubosidad y la precipitación. A pesar de reconocer que se puede dar una contaminación térmica, producto de los espejos de agua poco profundos propios de los sistemas de regadío, el daño ocasionado, al fin y al cabo, dependerá de la temperatura que pueda alcanzar el agua.

La estrategia para asignar un puntaje a las diferentes temperaturas medidas (Cuadro 1), se basó en la temperatura promedio del agua en el humedal La Bocana, ecosistema tropical a proteger, durante el tiempo de registro (julio 2002-abril 2004) que fue de $27.2 \pm 1.2{ }^{\circ} \mathrm{C}$, condición a la que las especies propias del lugar están adaptadas. Y en la máxima variación en la temperatura diaria obtenida durante cinco días, que correspondió a $1.4^{\circ} \mathrm{C}$. Este factor, permitió evaluar el alejamiento de la temperatura del valor ideal para la biota y por lo tanto, definir las categorías de calidad.

Desde la perspectiva planteada, las temperaturas dentro del ámbito $27.2 \pm 1.4{ }^{\circ} \mathrm{C}$, corresponden a una condición normal para las lagunas de inundación, razón por la cual se les asigna la categoría de excelente.

El doble de la máxima variación diaria de la temperatura marca a los valores $30.0{ }^{\circ} \mathrm{C}$ y $24.4{ }^{\circ} \mathrm{C}$, como los límites hasta los cuales la temperatura se asocia con una condición buena para el ecosistema. Esta decisión se tomó por su cercanía al parámetro de perturbación máxima aceptable durante 12 horas, establecido en $3^{\circ} \mathrm{C}$, cuando se da a lo largo de un período de 4 horas (Murphy 2002).

Una alteración de cinco veces la máxima variación diaria se consideró un estado no conveniente, por lo que las temperaturas $20.2{ }^{\circ} \mathrm{C}$ y $34.2{ }^{\circ} \mathrm{C}$ son los valores a partir de los cuales el agua se clasifica como mala. Además, se escogió una temperatura de $37^{\circ} \mathrm{C}$, diez grados por encima del valor óptimo, como el límite a partir del cual el agua se denota como no apta para la vida acuática.

Los ámbitos propuestos guardan relación con la comunidad biótica de la parte baja del río Mississippi. Allí, un aumento de 3,7 y $10^{\circ} \mathrm{C}$ sobre la temperatura preferida, permite que 
cerca del $90 \%, 55 \%$ y $25 \%$ de las especies, respectivamente, permanezcan dentro del ámbito de condición óptima (Bush et al. 1974).

Fósforo total: El fósforo es un componente esencial del ciclo biológico en los cuerpos de agua y generalmente, es el agente limitante del crecimiento de las algas y plantas acuáticas en humedales de agua dulce, por lo que su concentración sirve de criterio para reconocer un problema de eutrofización de lagos, lagunas o ríos y para definir el estado eutrófico de un cuerpo de agua (USDA 1999). Aunque, en lagos de zonas tropicales donde predominan las macrófitas, tiene limitaciones si se considera como criterio único (Parinet et al 2004).

En áreas agrícolas, el arrastre del fósforo ligado a las partículas erosionadas de los suelos (introducido por la aplicación de fertilizantes que contienen ortofosfatos) es la principal fuente de contaminación. Este efecto se incrementa con ciertas prácticas como las quemas y el fangueo.

En las lagunas de inundación de agua dulce, existen mecanismos para atrapar y reciclar el fósforo orgánico y el inorgánico, asociados a las actividades metabólicas. Este elemento puede ser introducido al cuerpo de agua por la acción bacteriana o cambios en el pH. En condiciones aerobias, se adsorbe o precipita por la formación de complejos insolubles con las formas oxidadas de hierro (III), calcio y aluminio. Mientras que en un entorno anaerobio y ácido, como el que normalmente se encuentra cerca de la capa de sedimentos, una buena parte del fósforo se convierte en una especie soluble, que difunde y pasa a ser utilizable por las plantas (Kadlec y Knight 1996). Por lo tanto, el contenido de fósforo total, da un estimado del fósforo potencialmente disponible y es la determinación analítica del elemento que mejor se ajusta a la calidad del agua (Parinet et al 2004).

Los ámbitos definidos para el fósforo en el Cuadro 1 se construyeron con base en concentraciones límite, establecidas a partir de criterios de sostenibilidad ambiental y de la información que se detalla a continuación.
Excelente, si una corriente de agua tiene concentraciones menores a $50 \mu \mathrm{g} \mathrm{P}_{\text {Total }} / 1$. Esta magnitud fue usada en el desarrollo del ICAVA, para definir el agua como muy buena y apta para la vida acuática (Rodríguez-Ulloa 1996). Además, este parámetro cumple con la restricción impuesta por algunos límites de cuantificación durante el trabajo de campo en el SRT donde se aplicó el índice.

De regular a mala, cuando el fósforo total presenta valores de $51 \mu \mathrm{g} / \mathrm{l}$ a $100 \mu \mathrm{g} / \mathrm{l}$. El criterio se estableció con base en los límites recomendados por la EPA para las aguas de vertido. Según éstos las corrientes al desaguar en un lago o reservorio no deben exceder los 50 $\mu \mathrm{g} / \mathrm{l}$, aquellas que no descargan directamente en estos ecosistemas no han de superar los 100 $\mu \mathrm{g} / \mathrm{l}$ y a partir de $100 \mu \mathrm{g} / \mathrm{l}$ de fósforo total, un reservorio se considera en estado hipereutrófico (EPA 2000).

Muy mala, de $101 \mu \mathrm{g} / 1$ a $150 \mu \mathrm{g} / 1$ de fósforo total. El límite superior marca el valor a partir del cual Rodríguez-Ulloa (1996) clasificó el agua como no apta para la vida acuática.

Demanda química de oxígeno: Es un indicador de contaminación orgánica, aporta básicamente la misma información que la $\mathrm{DBO}$, pero su análisis no requiere inocular las muestras tomadas en ambientes cercanos a anoxia. La diferencia entre los valores de DQO y DBO se origina primordialmente en la estabilidad de los ácidos fúlvicos y húmicos, los que aumentan la DQO pues sólo se oxidan en presencia de dicromato. Aunque sus concentraciones dependen de las características físico-geográficas, en condiciones naturales los ácidos húmicos y fúlvicos llegan a constituir hasta $80 \%$ de la DQO (Waite 1984).

En las lagunas de inundación el oxígeno disuelto puede no guardar relación con la demanda de oxígeno, al encontrarse en concentraciones bajas, sin que esto refleje un ingreso de contaminantes. Por ello, se incluyeron ambas variables en el ICA-L.

Los ámbitos de calidad para la DQO del Cuadro 1 se establecieron con base en criterios de sostenibilidad ambiental y la información disponible, según se detalla a continuación. 
Una demanda química máxima de $25 \mathrm{mg} \mathrm{O}_{2} / 1$, para caracterizar el agua como excelente, se fijó en concordancia con el límite de cuantificación de los análisis efectuados y el criterio de que este resultado se asocia a agua no contaminada. El intervalo de 26 a $40 \mathrm{mg}$ $\mathrm{O}_{2} / 1$, corresponde a una DQO apta para el desarrollo de la piscicultura y, el puntaje de calidad asignado al límite entre el agua de condición regular y mala $\left(40 \mathrm{mg} \mathrm{O}_{2} / \mathrm{l}\right)$ es el presentado por Escribano y De Frutos (1987).

El valor de $151 \mathrm{mg} \mathrm{O}_{2} / 1$ de DQO con una DBO mayor a $15 \mathrm{mg} \mathrm{O}_{2} / 1$, se seleccionó al considerar que establece una contaminación de materia orgánica tal, que vuelve el agua inapropiada para el funcionamiento del humedal.

La decisión de utilizar la función de calificación para calcular el puntaje de la DQO solo si la DBO es superior a los $2 \mathrm{mg} \mathrm{O} / 1$, obedece a que valores de DBO menores identifican aguas muy limpias, con muy poco material biodegradable. Por lo tanto, si los resultados aparecieran alterados, sería por la presencia de ácidos húmicos y fúlvicos, que no ejercen repercusiones importantes en la contaminación orgánica y la deflexión del oxígeno disuelto.

Agentes tóxicos: En estudios ambientales es importante cuantificar y definir el límite máximo permitido para aquellos contaminantes que según las posibles fuentes se perciban como una amenaza, especialmente tratándose de metales, por no haber mecanismos de eliminación natural.

En el caso específico del SRT, contaminantes de interés son el zinc, las grasas y los aceites, por estar asociados a las prácticas agrícolas del cultivo de arroz.

Aunque las sustancias tóxicas, como plaguicidas, grasas y aceites o metales pesados, no se incluyeron explícitamente en la ecuación del ICA-L, se contemplan mediante el criterio aceptable o inaceptable propuesto por el panel de expertos guiado por la National Sanitation Foundation (NSF) (Deininger 1980).

Zinc: El efecto perjudicial lo causa el ion $\mathrm{Zn}^{2+}$ en disolución, cuya concentración se relaciona directamente con la del zinc total, más fácil de medir. La toxicidad del $\mathrm{Zn}^{2+}$ se origina cuando éste desplaza los iones calcio o magnesio y bloquea su acción catalítica, o bien, modifica estructuras celulares vitales. Los iones $\mathrm{Ca}^{2+}(\mathrm{ac})$ $\mathrm{y} \mathrm{Mg}^{2+}$ (ac) evitan que el $\mathrm{Zn}^{2+}$ (ac) sea absorbido por los organismos acuáticos (SSRH 2004), así a mayor dureza menor es la toxicidad del metal y por ello, el criterio de rechazo del zinc se adaptó a la dureza de las aguas del DRAT.

Las funciones para calcular los límites permitidos para el Zn, relacionados con la preservación de la vida acuática, según la dureza del agua, fueron las establecidas por la EPA (2002). En el DRAT, donde la dureza promedio de las aguas fue de $80 \pm 36 \mathrm{mg} \mathrm{CaCO} / 1$ durante la época de muestreo, los criterios de rechazo del zinc resultaron ser: $99.3 \mu \mathrm{g} / \mathrm{l}$, concentración máxima de zinc total; $97.1 \mu \mathrm{g} / \mathrm{l}$, concentración máxima aguda de $\mathrm{Zn}^{2+}$ (ac) y $97.9 \mu \mathrm{g} / 1$, concentración continua de $\mathrm{Zn}^{2+}$ (ac).

Grasas y aceites: Son los contaminantes que han originado los mayores daños ecológicos, al causar problemas tales como: pérdida de movilidad en las aves acuáticas, asfixia en los peces y en formas de vida bentónica y mala apariencia del agua (EPA 1986).

El principal problema para fijar los valores de rechazo en este grupo surge de la cantidad de compuestos que engloba. Se han identificado más de 800 compuestos, con diferencias significativas en sus efectos ambientales. Las concentraciones máximas permitidas para el agua de consumo y la protección de la pesca están generalmente entre 0.01 y $0.1 \mathrm{mg} / \mathrm{l}$. Concentraciones de $0.3 \mathrm{mg} / 1$ o más de aceite crudo causan efectos tóxicos en los peces de agua dulce (Chapman 1996).

Al no existir límites definidos, se estimó como valor representativo deseable de rechazo $0.1 \mathrm{mg} / 1$ de grasas y aceites, concentración dentro del ámbito en que inician los problemas para las especies más sensibles, según los efectos en la biota presentados por EPA (1986).

El índice, su cálculo y aplicación: El ICA-NSF se seleccionó como punto de partida 
para el ICA-L porque ensayos de campo indican que es confiable; incluye varias de las propiedades que usualmente se estudian y, al ser muy utilizado facilita, aunque sea de manera parcial, futuras comparaciones con otras regiones. (Deininger 1980, Kahler-Royer 1999, Swamee y Tyagi 2007).

En el cálculo del ICA-L se utiliza el producto ponderado porque evita el eclipsamiento (Couillard y Lefebvre 1985) y concuerda mejor, en ensayos de validación, con la valoración de un juego de muestras individuales realizada por expertos (Deininger 1980).

El ICA-L construido permite evaluar la capacidad del agua de las lagunas tropicales de inundación de agua dulce, para lograr el sostenimiento de la biodiversidad y el desarrollo de la vida acuática, cuando los peligros surgen por prácticas agropecuarias. Esto es debido a la incorporación de factores específicos como: la puntuación asignada al porcentaje de saturación del oxígeno disuelto no superficial en lagunas; la calificación de la temperatura, la construcción del puntaje para la concentración de fósforo total con base en los límites recomendados por la EPA para las corrientes que desaguan en un lago o reservorio y, la alta ponderación de variables como los sólidos suspendidos, mientras no se incluyen los sólidos sedimentables y los coliformes fecales.

Los agentes tóxicos prioritarios y los límites máximos permitidos para el zinc se ajustaron a las condiciones de las lagunas del PNPV y los regadíos colindantes. Sin embargo, este aspecto no modifica el interés por lograr un índice de aplicación general, para definir la calidad del agua en lagunas de inundación; pues el criterio de fijar en cero el índice si se sobrepasan los límites permitidos para los agentes tóxicos, lo deja abierto a las particularidades de cada ecosistema.

\section{AGRADECIMIENTOS}

A la Fundación Costa Rica-Estados Unidos para la Cooperación, a la Organización para Estudios Tropicales (OET) y al Centro de Investigación en Contaminación Ambiental
(CICA) de la Universidad de Costa Rica, por el financiamiento aportado a este proyecto. A las siguientes personas Eugenio González y Jenaro Acuña, por sus oportunas sugerencias y correcciones. A todo el personal de la Estación Biológica Palo Verde y de la OET, particularmente a: Francisco Enríquez, Oscar Arias, Mauricio Castillo, Manuel Blázquez y Marcela Rivera. A Elizabeth Carazo y a todo el personal del CICA, en especial a Katia Villalobos, Desireé Sauma y Edipcia Roque por su apoyo en el análisis de las muestras. Y al Servicio Nacional de Riego y Avenamiento (SENARA) por su colaboración.

\section{RESUMEN}

Se creó un índice fisicoquímico de calidad del agua (ICA-L), para lagunas que se desbordan, el cual fue validado en el sector de riego de Tamarindo, y en una sección del sistema de lagunas del Parque Nacional Palo Verde (Guanacaste, Costa Rica). El índice incluye las variables: porcentaje de saturación de oxígeno disuelto, $\mathrm{pH}$, concentración de nitratos, concentración de fósforo total, demanda química de oxígeno, concentración de sólidos suspendidos, conductividad eléctrica y temperatura. El índice se fija automáticamente en cero si la concentración de alguna sustancia tóxica excede el máximo permitido. Los factores de ponderación se ajustaron con base en los pesos definidos en el Índice de Calidad de Agua de la National Sanitation Foundation (ICA-NSF), se excluyó el peso del conteo de coliformes fecales, se integró la ponderación de turbiedad y de sólidos totales una sola, de sólidos suspendidos, y se asignó a la conductividad un factor de 0.08 . El índice permite evaluar la capacidad del agua de las lagunas tropicales de inundación de agua dulce, para lograr el sostenimiento de la biodiversidad y el desarrollo de la vida acuática, cuando surgen peligros por prácticas agropecuarias.

Key words: índice de calidad del agua, sistema tropical de agua dulce, humedal, laguna estacional, índice físicoquímico, Palo Verde.

\section{REFERENCIAS}

Alberti, M. \& J. Parker. 1991. Indices of environmental quality: the search for credible measures. Environ. Impact Assess. Rev. 11: 95-101.

Astorga, Y. 1994. Informe final: Diagnóstico fisicoquímico y biológico de la quebrada Los Negritos. Centro de Investigación en Contaminación Ambiental, 
Universidad de Costa Rica, San José, Costa Rica. $32 \mathrm{p}$.

Boyacioglu, H. 2007. Development of water quality index based on of European classification scheme. Water. 33: 101-106.

Bush, R.M, E.B. Welch \& B.W. Mar. 1974. Potential effects of thermal discharges on acuatic systems. Environ. Sci. Technol. 8: 561-568.

Chapman, D. 1996. Water quality assessments: A guide to the use of biota, sediments and water in environmental monitoring. E\& FN Spon, Londres, Inglaterra. 626 p.

Couillard, D. \& Y. Lefebvre. 1985. Analysis of water quality index. J. Environ. Manag. 21: 161-179.

Cude, C. 2001. Oregon water quality index: a tool for evaluating water quality management effectiveness. JAWRA. 37: 125-137.

Dagne, D., W. Owens \& P. Tchounwou. 2005. Comparative assessment of the physico-chemical and bacteriological qualities of selected streams in Louisiana. Int. J. Environ. Res. Public Health. 2: 94-100.

Deininger, R.A. 1980. A water quality index for rivers. Wat. Int. September: 16-21.

Escribano, M. \& M. De Frutos. 1987. Impactos sobre la calidad de las aguas superficiales, p. 443-453. In Escuela Técnica Superior de Ingenieros de Montes (eds.). La práctica de las estimaciones de impactos ambientales, tomo 3. Fundación Conde del Valle de Salazar, Madrid, España.

EPA. 1986. Quality criteria for water. Office of Water Regulations and Standards, U.S. Environmental Protection Agency, Washington DC, EEUU. EPA 440/5-86-001. 477 p.

EPA. 2000. Nutrient criteria technical guidance manuallakes and reservories. Office of Water \& Office of Science and Technology, U.S. Environmental Protection Agency, Washington DC, EEUU. EPA822-B00-001. 228 p.

EPA. 2002. National recommended water quality criteria. Office of Water \& Office of Science and Technology, U.S. Environmental Protection Agency, Washington DC, EEUU. EPA-882-R-02-047. 36 p.

Figueroa, R., A. Palma, V. Ruiz \& X. Niell. 2007. Análisis comparativo de índices bióticos utilizados en la evaluación de la calidad de las aguas en un río mediterráneo de Chile: río Chillan, VIII región. Rev. Chil. Hist. Nat. 80: 225-242.
Fuentes, F. \& A. Massol-Deyá. 2002. Manual de laboratorios: Ecología de microorganismos. Universidad de Puerto Rico, Puerto Rico, EEUU. 265 p.

García, M., F. Darío, R. Marín, H. Guzmán, N. Verdugo, E. Domínguez, O. Vargas, L. Panizzo, N. Sánchez, J. Gómez \& G. Cortés. 2001. El agua, p.114-407. In P. Leyva (ed). El ambiente en Colombia. Instituto de hidrología, meteorología y estudios ambientales, IDEAM, Bogotá, Colombia. 543 p.

Gaunt, P. \& S. Barker. 2000. Matrix solid phase dispersion extraction of triazines from catfish tissues; examination of the effects of temperature and dissolved oxygen on the toxicity of atrazine. Int. J. Environ. Pollut. 13: 284-312.

Horn, S.P. \& K.A. Habeyran. 1993. Physical and chemical properties of Costa Rican lakes. Natl. Geogr. Res. Explor. 9: 86-103.

House, M.H. 1990. Water quality indices as indicators of ecosystem change. Environ. Monit. Assess. 15: 255-263.

Kadlec, R.H. \& R.L. Knight. 1996. Treatment Wetlands. CRC, Boca Ratón, Florida, EEUU. 893 p.

Kahler-Royer, C.A. 1999. A water quality index devised for the Des Moines River in Central Iowa. Tesis de maestría, Iowa State University, Ames, Iowa. 168 p.

Lampert, W. \& U. Sommer. 1997. Limnoecology: The ecology of lakes and streams. Oxford University Press, Nueva York, EEUU. 382 p.

Parinet, B., A. Lhote \& B. Legube. 2004. Principal component analysis: an appropriate tool for water quality evaluation and management - application to a tropical lake system. Ecol. Model. 178: 295-311.

Primavesi, O., A. Ribeiro de Freitas, A. Primavesi \& H. Torres de Oliveira. 2002. Water quality of the Canchim's Creek watershed in São Carlos, SP, Brazil, occupied by beef and dairy cattle activities. Braz. Arch. Biol. Technol. 45: 209 - 217.

Ramsar. 1998. Procedimiento de orientación para la gestión. Informe $\mathrm{N}^{\circ}$ 39: Sitio Ramsar Parque Nacional Palo Verde, Costa Rica. Oficina de la Convención sobre los Humedales, Gland, Suiza.

Rickert, D. 1993. Evaluación de la calidad del agua para determinar la naturaleza y el grado de contaminación del agua por la agricultura y actividades afines, p.187-209. In Prevención de la contaminación del agua por la agricultura y actividades afines. FAO, Santiago de Chile, Chile. 385 p. 
Rizo-Patrón, F. 2003. Estudio de los arrozales del proyecto Tamarindo: Agroquímicos y macroinvertebrados bentónicos en relación al Parque Nacional Palo Verde, Guanacaste, Costa Rica. Tesis de maestría, Universidad Nacional, Heredia, Costa Rica. 118 p.

Rodríguez, R.A. 1995. Humedal: Sistema de tratamiento de aguas residuales. Tesis de licenciatura, Universidad de Costa Rica, San José, Costa Rica. 107 p.

Rodríguez-Ulloa, A. 1996. Informe Final: Vigilancia de la calidad de las aguas superficiales en la zona de influencia del Proyecto de riego Arenal Tempisque. Centro de investigación en Contaminación Ambiental, Universidad de Costa Rica, San José, Costa Rica. 38 p.

Said, A., D. Stevens \& G. Sehlke. 2004. A innovative water quality index. Environ. Manag. 34:406-414.

SSRH. 2004. Nivel guía de calidad de agua ambiente para protección de la biota acuática correspondiente a zinc, p III.1-III.2. In Desarrollos de niveles guía nacionales de calidad de agua ambiente correspondientes a zinc. Subsecretaría de Recursos Hídricos de la Nación, Buenos Aires, Argentina.
Swamee, P.K. \& A. Tyagi. 2007. Improved method for aggregation of water quality subindices. J.Environ. Eng. 133: 220-225.

UICN. 2002. Seguimiento de las directrices de la Convención RAMSAR en la planificación de los humedales de importancia internacional en Centroamérica. Unión Mundial para la Naturaleza, Oficina regional para Mesoamérica, Gobierno de Noruega, San José, Costa Rica. 72 p.

USDA. 1999. A procedure to estimate the response of aquatic systems to changes in phosphorus and nitrogen inputs. U.S. Department of Agriculture, Natural Resources Conservation Service, Washington DC, EEUU. 34 p.

Waite, T. 1984. Principles of water quality. Academic, Orlando, Florida, EEUU. 289 p.

\section{REFERENCIAS DE INTERNET}

Murphy, S. 2007. General information on temperature. USGS Water Quality Monitoring, Boulder, Colorado, EEUU. (Consultado: mayo 22, 2006, http://bcn.boulder.co.us/basin/data/COBWQ/info/Temp.html). 